\title{
Performance of DS-CDMA Downlink Using Transmitter Preprocessing and Relay Diversity over Nakagami- $m$ Fading Channels
}

\author{
Wei Fang, Student Member, IEEE, Lie-Liang Yang, Senior Member, IEEE, and Lajos Hanzo, Fellow, IEEE
}

\begin{abstract}
In this letter we propose and investigate a cooperative downlink transmission scheme for direct-sequence codedivision multiple-access (DS-CDMA) systems, in order to achieve relay diversity. Transmitter zero-forcing (TZF)-based preprocessing is employed at the base-station (BS) of our proposed scheme for suppressing the downlink multiuser interference (MUI). At the mobile terminals (MTs) the signals are combined based on the maximal ratio combining (MRC) principles. As a benefit of TZF, the BS decontaminates the relayed signals from MUI without requiring any information from the relays. Furthermore, only low-complexity signal processing is necessary at the relays for forwarding information to the destination MTs (DMTs), while achieving the relay diversity by only exploiting the knowledge of the DMTs' spreading sequences.
\end{abstract}

Index Terms-Transmitter preprocessing, zero-forcing, relay diversity, DS-CDMA, cooperation.

\section{INTRODUCTION}

$\mathbf{R}$ ECENTLY, cooperative diversity aided wireless systems have attracted substantial research attention $[1,2]$. In a wireless system geographically distributed MTs may share their antennas in order to create a virtual multiple transmit antenna aided system. It has been shown that the capacity or error performance of a wireless system may be significantly improved by the cooperation of the distributed MTs [1-5]. Specifically, in a DS-CDMA downlink supporting multiple MTs (referred to as destination MTs or DMTs), a number of MTs (referred to as relays) close to a DMT may be chosen to assist its downlink reception in order to achieve relay diversity. However, the multiuser DS-CDMA downlink signals may interfere with each other, generating multiuser interference (MUI). In this case, the relay diversity may not be achieved without suppressing the MUI [6].

In this contribution, we propose and investigate a cooperative DS-CDMA downlink transmission scheme. In our proposed scheme the downlink MUI imposed on both the relays and DMTs is suppressed with the aid of transmitter zeroforcing (TZF)-assisted preprocessing operated at the BS [710]. The BS carries out TZF-assisted preprocessing without requiring any information from the relays. Furthermore, it does not require any channel state information (CSI) from the DMTs, except for the knowledge of their spreading sequences. At the DMTs, the signals received from both the BS and the relays are combined based on the MRC principles [12]. Our study and simulation results demonstrate that the proposed

Manuscript received April 8, 2008; revised July 23, 2008 and September 4, 2008; accepted September 19, 2008. The associate editor coordinating the review of this paper and approving it for publication was M. Tao.

The authors are with the School of Electronics and Computer Science, University of Southampton, SO17 1BJ, United Kingdom, (e-mail: \{wf04r, 1ly, lh\}@ecs.soton.ac.uk).

Digital Object Identifier 10.1109/TWC.2009.080479

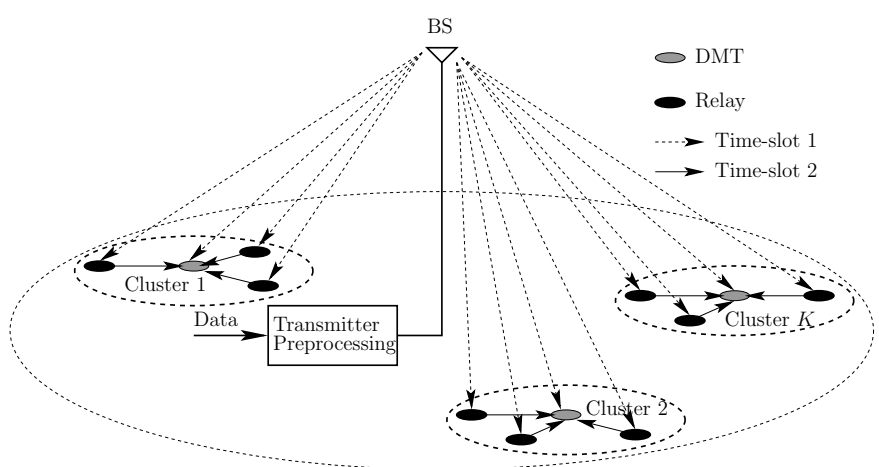

Fig. 1. Schematic block diagram of the relay-aided DS-CDMA downlink system using zero-forcing (ZF) assisted transmitter preprocessing to support $K$ mobile terminals. Each of the $K$ mobile terminals is assisted by $L$ relays for achieving a $L$ th order relay diversity.

cooperative downlink transmission scheme is capable of efficiently mitigating the MUI and of achieving relay diversity.

The rest of this letter is organised as follows. Section II details the operations of the DS-CDMA downlink, including the transmitted signal and transmitter preprocessing. Section III considers the operation of the relays. In Section IV the detection at the BS is discussed. Section V presents our simulation results, and finally, in Section VI we provide our conclusions.

\section{SYSTEM DESCRIPTION}

\section{A. Transmitted Signal and Assumptions}

Let us consider the DS-CDMA downlink transmitting to $K$ DMTs (or users), as shown in Fig. 1, where each DMT is supported by $L$ relays, which are constituted by the MTs without their own data to transmit/receive. For convenience, we define the direct (D)-channels as those directly connecting (via wireless) the BS with the DMTs, and the relay (R)channels as the channels connecting the BS with the DMTs through relays. Furthermore, the R-channels are divided into the BR-channels spanning the BS to the relays and the RMchannels linking the relays to the DMTs.

Throughout this paper we assume that the cooperation operation is based on time-division (TD). Specifically, in the cooperation scheme considered a symbol duration is divided into two time-slots (TSs). The BS processes and broadcasts information synchronously to the $K$ DMTs and also to the $K L$ number of relays using the first TS. During the second TS, the $K L$ relays forward the information received within the first TS also synchronously to their corresponding DMTs. In this contribution we assume that a DMT and its relays form a cooperating cluster. We assume for simplicity that any two 
of the clusters are sufficiently separated, so that each cluster may be deemed free from interference during the second TS, after taking into account the distance-dependent propagation pathloss. Note that this assumption is applied only for the sake of simplicity. In fact, our study can be directly applied to the scenarios eliminating the above-mentioned assumption, when the inter-cluster interference is approximated by Gaussian noise. It can also be readily generalized to scenarios contaminated by inter-cluster interference.

Let $s$ denote the $N$-length signal vector to be transmitted to the $K$ DMTs over the DS-CDMA downlink, where $K \leq N$ is assumed, since TZF-assisted linear transmitter preprocessing is employed. When considering both the DS-spreading and transmitter preprocessing, $s$ can be expressed as

$$
s=P C b
$$

where $\boldsymbol{P}$ is a $(N \times N)$-dimensional transmitter preprocessing matrix and $C$ is a $(N \times K)$-dimensional DS-spreading matrix containing the spreading sequences assigned to the $K$ DMTs, which is given by

$$
\boldsymbol{C}=\left[\boldsymbol{c}_{1}, \boldsymbol{c}_{2}, \cdots, \boldsymbol{c}_{K}\right]
$$

where the $k$ th DMT's spreading sequence is expressed as

$$
\boldsymbol{c}_{k}=\left[c_{k 0}, c_{k 1}, \cdots, c_{k(N-1)}\right]^{T}, k=1,2, \cdots, K
$$

and $\boldsymbol{c}_{k}$ is normalized to satisfy $\boldsymbol{c}_{k}^{T} \boldsymbol{c}_{k}=1$. Finally, in (1) $\boldsymbol{b}$ denotes a $K$-length vector of

$$
\boldsymbol{b}=\left[b_{1}, b_{2}, \cdots, b_{K}\right]^{T}
$$

which contains the $K$ symbols to be transmitted to the $K$ DMTs. It is assumed that $b_{k}$ obeys $E\left[\left|b_{k}\right|^{2}\right]=1$.

We assume that the downlink channels experience flat fading. Then, it can be shown that after the chip-level matchedfiltering, the observation vector obtained at the $k$ th DMT can be expressed as

$$
\boldsymbol{r}_{k}=h_{k} \boldsymbol{P C b}+\boldsymbol{n}_{k}, \quad k=1,2, \ldots, K
$$

where $h_{k}$ represents the complex-valued channel gain, while $\boldsymbol{n}_{k}$ is a $N$-length Gaussian noise vector, which obeys the multivariate Gaussian distribution with zero mean and a covariance matrix of $2 \sigma^{2} \boldsymbol{I}_{N}$, where $\sigma^{2}=1 / 2 \mathrm{SNR}$ and SNR represents the signal-to-noise ratio.

After despreading at DMT $k$ using its spreading sequence $\boldsymbol{c}_{k}$, the decision variable for $b_{k}$ can be expressed as

$$
y_{k}=\boldsymbol{c}_{k}^{T} \boldsymbol{r}_{k}=h_{k} \boldsymbol{c}_{k}^{T} \boldsymbol{P} \boldsymbol{C b}+n_{k}, k=1,2, \ldots, K
$$

where $n_{k}=\boldsymbol{c}_{k}^{T} \boldsymbol{n}_{k}$, which is a Gaussian random variable distributed with zero mean and a variance of $\sigma^{2}$ per dimension.

Let us collect the decision variables of the $K$ DMTs into $\boldsymbol{y}=\left[y_{1}, y_{2}, \ldots, y_{K}\right]^{T}$. Then, it can be shown that we have

$$
y=H C^{T} P C b+n
$$

where $\boldsymbol{H}=\operatorname{diag}\left\{h_{1}, h_{2}, \ldots, h_{K}\right\}$ and $\boldsymbol{n}=\left[n_{1}, n_{2}, \ldots, n_{K}\right]^{T}$. Let us now consider the transmitter preprocessing.

\section{B. Transmitter Zero-Forcing Preprocessing}

The MUI among the $K$ DMTs is suppressed based on the TZF preprocessing principles by exploiting only the knowledge about the spreading sequences of the $K$ DMTs at the BS [7,9-11]. More explicitly, the preprocessing does not make use of any knowledge about the fading channels, ie., $\left\{h_{k}\right\}$, and the reason for this will become explicit in our forthcoming discourse, when we discuss the cooperation philosophy in Section III. Based on (1) and (7), we can know that both spreading and transmitter preprocessing can be implemented simultaneously in a single step. Let express $\tilde{\boldsymbol{P}}=\boldsymbol{P C}=\left[\tilde{\boldsymbol{p}}_{1}, \tilde{\boldsymbol{p}}_{2}, \cdots, \tilde{\boldsymbol{p}}_{K}\right]$, where $\tilde{\boldsymbol{p}}_{k}$ is for joint spreading and preprocessing of $b_{k}$. Then, when the TZF-assisted preprocessing is considered, the spreading/preprocessing matrix $\tilde{\boldsymbol{P}}$ can be chosen as [7,9-11]

$$
\tilde{P}=\beta C\left(\boldsymbol{C}^{T} \boldsymbol{C}\right)^{-1}
$$

where the parameter $\beta$ is chosen to satisfy the power constraint of $E\left[\|\tilde{\boldsymbol{P}} \boldsymbol{b}\|^{2}\right]=E\left[\|\boldsymbol{b}\|^{2}\right]=K$, yielding

$$
\beta=\sqrt{\frac{K}{\operatorname{trace}\left(\left(\boldsymbol{C}^{T} \boldsymbol{C}\right)^{-1}\right)}} .
$$

After inserting (8) into (7), the decision variables corresponding to the $K$ downlink DMTs can be expressed as

$$
\boldsymbol{y}=\beta \boldsymbol{H} \boldsymbol{b}+\boldsymbol{n}
$$

Explicitly, the MUI among the $K$ downlink DMTs is perfectly removed by the TZF-assisted preprocessing. However, as always [7,9-11] the TZF-assisted preprocessing removes the downlink MUI at the cost of noise amplification.

\section{CoOperation Operations AT RElays}

So far, the operations at the relays have not been considered in our discussions, since there are no communications between the BS and relays as the BS has no knowledge about the relays. Let us now consider the operations at the relays. When the signal of (1) formed with the aid of the spreading/preprocessing matrix of (8) is broadcast on the downlink, it can be shown that the observation vector obtained by the $l$ th relay of the $k$ th DMT within the first TS of a symbol duration can be expressed as

$$
\boldsymbol{r}_{l}^{(k)}=h_{l}^{(k)} \tilde{\boldsymbol{P}} \boldsymbol{b}+\boldsymbol{n}_{l}^{(k)}, l=1,2, \ldots, L
$$

where $\boldsymbol{n}_{l}^{(k)}=\left[n_{l 0}^{(k)}, n_{l 1}^{(k)}, \cdots, n_{l(N-1)}^{(k)}\right]^{T}$ denotes the $N$ length Gaussian noise vector, which obeys the complex multivariate Gaussian distribution with zero mean and a covariance matrix of $2 \sigma^{2} \boldsymbol{I}_{N}$, while $h_{l}^{(k)}$ represents the complex-valued channel gain of the BR-channel spanning from the BS to the $l$ th relay of DMT $k$.

We assume that the relays of DMT $k$ employ the knowledge of $\boldsymbol{c}_{k}$ and that the $l$ th relay of DMT $k$ is capable of acquiring the knowledge of $h_{l}^{(k)}$. Then, despreading $\boldsymbol{r}_{l}^{(k)}$ of (11) using $c_{k}$ gives

$$
y_{l}^{(k)}=h_{l}^{(k)} \boldsymbol{c}_{k}^{T} \tilde{\boldsymbol{P}} \boldsymbol{b}+\boldsymbol{c}_{k}^{T} \boldsymbol{n}_{l}^{(k)}=\beta h_{l}^{(k)} b_{k}+n_{l}^{(k)}
$$

where $n_{l}^{(k)}=\boldsymbol{c}_{k}^{T} \boldsymbol{n}_{l}^{(k)}$. Equation (12) shows that the reception at the relays is free from any downlink MUI. Furthermore, 
given the knowledge of $h_{l}^{(k)}$, the soft estimate $\hat{b}_{l}^{(k)}$ of $b_{k}$ may be formed as

$$
\hat{b}_{l}^{(k)}=\beta b_{k}+\frac{1}{h_{l}^{(k)}} \boldsymbol{c}_{k}^{T} \boldsymbol{n}_{l}^{(k)}
$$

Based on the estimate of $\hat{b}_{l}^{(k)}$ in (13), $\hat{b}_{l}^{(k)}$ is re-spread using the unique spreading sequence assigned to the $l$ th relay of DMT $k$ and then forwarded to DMT $k$ using the second TS of a symbol. The signal vector transmitted by the $l$ th relay of DMT $k$ can be expressed as

$$
\boldsymbol{s}_{l}^{(k)}=\sqrt{\frac{1}{\varsigma_{k l}}} \boldsymbol{c}_{l}^{(k)} \hat{b}_{l}^{(k)}, l=1,2, \cdots, L
$$

where the power normalization factor $\varsigma_{k l}$ is given by

$$
\varsigma_{k l}=\mathrm{E}\left[\left|\hat{b}_{l}^{(k)}\right|^{2}\right]=\beta^{2}+\frac{2 \sigma^{2}}{\left|h_{l}^{(k)}\right|^{2}}
$$

Note that it is desirable to ensure that the spreading sequences assigned to the relays of the same cluster have low cross-correlation coefficients. However, the same spreading sequence may be assigned to several relays belonging to different clusters, which are sufficiently separated in space, resulting in that the inter-cluster interference is negligible.

From the above analysis, we can see that DMT $k$ can obtain two sets of observations from the first and second TSs of a symbol-duration, respectively, in order to detect the transmitted signals. Specifically, at DMT $k$, the observation vector obtained from the first TS of a symbol duration can be expressed as

$$
\boldsymbol{r}_{0}=h_{0}^{(k)} \tilde{\boldsymbol{P}} \boldsymbol{b}+\boldsymbol{n}_{0}
$$

where $h_{0}^{(k)}$ represents the complex-valued channel gain of the D-channel linking the BS with DMT $k$, while $\boldsymbol{n}_{0}$ denotes the $N$-length Gaussian noise vector.

By contrast, the observation vector obtained by DMT $k$ in the second TS of a symbol duration can be expressed as

$$
\begin{aligned}
\boldsymbol{r}_{1}= & \sum_{l=1}^{L} \sqrt{\frac{1}{\varsigma_{k l}}} h_{r l}^{(k)} \boldsymbol{c}_{l}^{(k)} \beta b_{k} \\
& +\sum_{l=1}^{L} \sqrt{\frac{1}{\varsigma_{k l}}} h_{r l}^{(k)} \boldsymbol{c}_{l}^{(k)}\left[\frac{\boldsymbol{c}_{k}^{T} \boldsymbol{n}_{l}^{(k)}}{h_{l}^{(k)}}\right]+\boldsymbol{n}_{1}
\end{aligned}
$$

where $h_{r l}^{(k)}$ denotes the complex-valued channel gain of the RM-channel linking the $l$ th relay of DMT $k$ with DMT $k$ and $\boldsymbol{n}_{1}$ denotes the $N$-length Gaussian noise vector. Note that both $\boldsymbol{n}_{0}$ in (16) and $\boldsymbol{n}_{1}$ in (17) are assumed to obey a common Gaussian distribution with zero mean and a covariance matrix of $2 \sigma^{2} \boldsymbol{I}_{N}$.

\section{Signal Detection at Mobile Terminals}

In this section we consider the signal detection at the DMTs. The signals received by a DMT from the first and second TSs are combined based on maximal ratio combining (MRC). From our discussions in Section II-B, we know that DMT $k$ encounters no MUI during the first TS due to the employment of the TZF-assisted preprocessing. Hence, after despreading $\boldsymbol{r}_{0}$ of (16) using $\boldsymbol{c}_{k}$, we obtain

$$
z_{0}=\boldsymbol{c}_{k}^{T} \boldsymbol{r}_{0}=h_{0}^{(k)} \beta b_{k}+n_{0}
$$

where $n_{0}=\boldsymbol{c}_{k}^{T} \boldsymbol{n}_{0}$. Furthermore, when MRC is considered, the weight used for combining $z_{0}$ can be expressed as

$$
w_{0}=\frac{\beta\left(h_{0}^{(k)}\right)^{*}}{2 \sigma^{2}}
$$

By contrast, the received signal vector $\boldsymbol{r}_{1}$ of (17) is despread using $\boldsymbol{C}_{k}=\left[\boldsymbol{c}_{1}^{(k)}, \boldsymbol{c}_{2}^{(k)}, \cdots, \boldsymbol{c}_{L}^{(k)}\right]$, which is constructed by the spreading codes of the relays supporting DMT $k$, yielding

$$
z=C_{k}^{T} \boldsymbol{r}_{1}
$$

where $z=\left[z_{1}, z_{2}, \cdots, z_{L}\right]^{T}$. Upon substituting (17) into (20), we can express the $l$ th, $l=1, \cdots, L$, component of $z$ as

$$
\begin{aligned}
z_{l}= & \sqrt{\frac{1}{\varsigma_{k l}}} h_{r l}^{(k)} \beta b_{k}+\left(\boldsymbol{c}_{l}^{(k)}\right)^{T} \boldsymbol{n}_{1} \\
& +\sum_{l^{\prime}=1}^{L} \sqrt{\frac{1}{\varsigma_{k l^{\prime}}}} \frac{h_{r l^{\prime}}^{(k)}}{h_{l^{\prime}}^{(k)}}\left(\boldsymbol{c}_{l}^{(k)}\right)^{T} \boldsymbol{c}_{l^{\prime}}^{(k)} \boldsymbol{c}_{k}^{T} \boldsymbol{n}_{l^{\prime}}^{(k)}+I_{R} \\
& l=1,2, \ldots, L
\end{aligned}
$$

where $I_{R}$ is the inter-relay interference imposed by the relays (except the $l$ th relay itself) of DMT $k$, which can be expressed as

$$
I_{R}=\sum_{l^{\prime}=1, l^{\prime} \neq l}^{L} \sqrt{\frac{1}{\varsigma_{k l}}} h_{r l^{\prime}}^{(k)}\left(\boldsymbol{c}_{l}^{(k)}\right)^{T} \boldsymbol{c}_{l^{\prime}}^{(k)} \beta b_{k}
$$

It can be shown that, when MRC is considered, $z_{l}$ for $l=$ $1,2, \ldots, L$ are approximated as independent Gaussian random variables. Correspondingly, the weights used for combining $\left\{z_{l}\right\}$ can be expressed as [6]

$$
\begin{aligned}
w_{l}= & \left(2 \sigma^{2} \sum_{l^{\prime}=1}^{L} \frac{\left|h_{r l^{\prime}}^{(k)}\right|^{2}}{\varsigma_{k l^{\prime}}\left|h_{l^{\prime}}^{(k)}\right|^{2}}\left|\left(\boldsymbol{c}_{l}^{(k)}\right)^{T} \boldsymbol{c}_{l^{\prime}}^{(k)}\right|^{2}+2 \sigma^{2}+2 \sigma_{R}^{2}\right)^{-1} \\
& \times \sqrt{\frac{1}{\varsigma_{k l}}} \beta\left(h_{r l}^{(k)}\right)^{*} \\
& l=1,2, \ldots, L
\end{aligned}
$$

where $2 \sigma_{R}^{2}$ is the second-order moment of $I_{R}$, which is given by

$$
2 \sigma_{R}^{2}=\sum_{l^{\prime}=1, l^{\prime} \neq l}^{L} \frac{1}{\varsigma_{k l^{\prime}}}\left|h_{r l^{\prime}}^{(k)}\left(\boldsymbol{c}_{l}^{(k)}\right)^{T} \boldsymbol{c}_{l^{\prime}}^{(k)} \beta\right|^{2}
$$

Finally, after taking into account the contributions from both the first and second TSs, the decision variable $z^{(k)}$ of $b_{k}$ can be formed according to the MRC principles as

$$
z^{(k)}=\sum_{l=0}^{L} w_{l} z_{l}
$$

where $w_{l}$ is given by (19) when $l=0$, and by (23), otherwise. Let us now provide our simulation results.

\section{Simulation Results}

In this section we provide two examples of simulation results in order to illustrate the BER performance of the DSCDMA downlink using the TZF-assisted preprocessing and relay diversity. In our simulations we assumed that binary phaseshift keying (BPSK) baseband modulation was employed. 


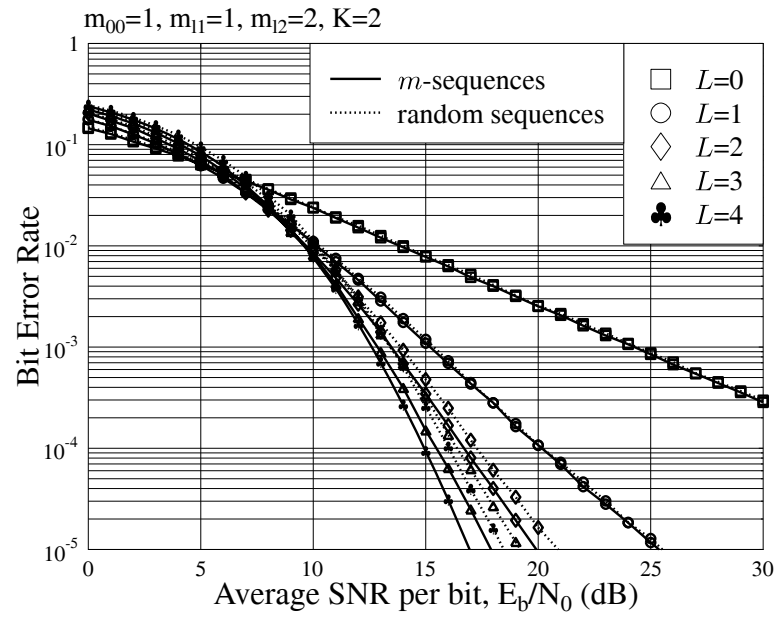

Fig. 2. BER versus SNR per bit performance of the relay-aided DS-CDMA downlink supporting $K=2$ DMTs, when the D-channel and BR-channels experience Rayleigh fading, while the RM-channels experience Nakagami- $m$ fading associated with the fading parameters of $m_{l 2}=2$ for $L=1,2,3,4$.

We assumed that the D-channels, BR-channels as well as the RM-channels might experience different fading modelled by the Nakagami- $m$ distributions associated with different values of the fading parameter $m$ [13]. Specifically, assign $h_{0}^{(k)}=\alpha_{00} e^{j \theta_{00}}$ in (16), $h_{l}^{(k)}=\alpha_{l 1} e^{j \theta_{l 1}}$ for $l=1, \ldots, L$ in (11), and $h_{r l}^{(k)}=\alpha_{l 2} e^{j \theta_{l 2}}$ for $l=1, \ldots, L$ in (17). Then, when Nakagami- $m$ fading channels are assumed, the probability density function (PDF) of $\alpha_{l i}$, where $i=0,1,2$ and $l=0,1, \ldots, L$, can be expressed as $[13,14]$

$$
\begin{array}{r}
f\left(\alpha_{l i}\right)=\frac{2 m_{l i}^{m_{l i}} \alpha_{l i}^{2 m_{l i}-1}}{\Gamma\left(m_{l i}\right) \Omega_{l i}} e^{-\left(m_{l i} / \Omega_{l i}\right) \alpha_{l i}^{2}}, \\
i=0,1,2 ; l=0,1, \ldots, L
\end{array}
$$

where $m_{l i}$ represents the fading parameter corresponding to the D-channel for $l=0, i=0$, to the $l$ th BR-channel for $l \geq 1, i=1$ or to the $l$ th RM-channel for $l \geq 1, i=2$. In (26) $\Omega_{l i}=E\left[\alpha_{l i}^{2}\right]$ represents the average power of the corresponding transmission link, which was assumed to be unity in our simulations. Furthermore, in our simulations the phases introduced by carrier modulation and fading channels were assumed to be independent identically distributed (iid) random variables uniformly distributed within $[0,2 \pi)$.

Figs. 2 and 3 show the BER versus the average SNR per bit $\left(E_{b} / N_{0}\right)$ performance of the relay-aided DS-CDMA downlink using the proposed TZF-assisted preprocessing, when assuming that the D-channel and BR-channels experience Rayleigh fading, while the RM-channels encounter Nakagami- $m$ fading associated with a fading parameter of $m_{l 2}=2$. Note that the reason for us to use different channel models for the Dchannels, BR-channels and the RM-channels is that we have assumed that the relays are close to their DMTs, but both the relays and DMTs are far away from the BS. In this case, the Dchannels and BR-channels may experience much more severe fading than the RM-channels. In our simulations the spreading factor of the DS-CDMA system was assumed to be $N=31$; both $m$-sequences and random sequences were considered. We assumed that the number of DMTs supported by the relay-

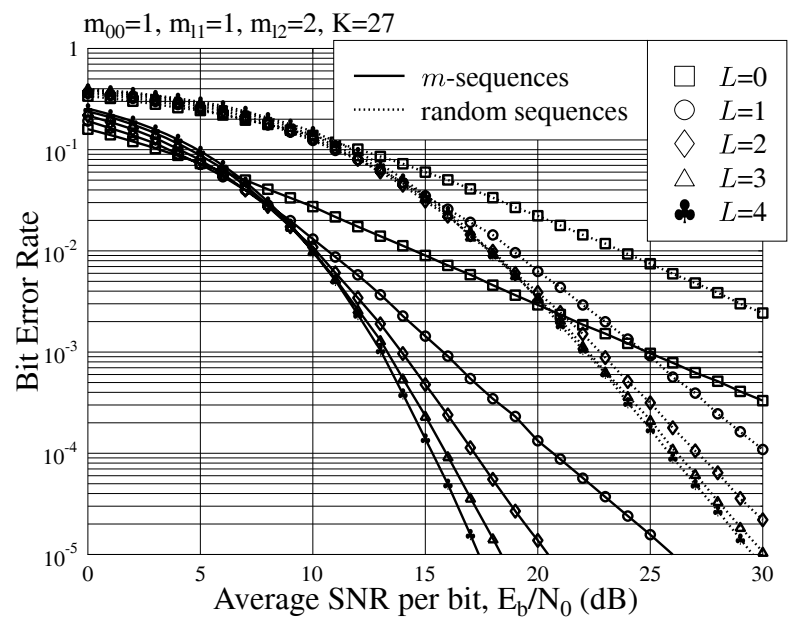

Fig. 3. BER versus SNR per bit performance of the relay-aided DS-CDMA downlink supporting $K=27$ DMTs, when the D-channel and BR-channels experience Rayleigh fading, while the RM-channels experience Nakagami- $m$ fading associated with the fading parameters of $m_{l 2}=2$ for $L=1,2,3,4$.

aided DS-CDMA downlink was $K=2$ for Fig. 2 and was $K=27$ for Fig. 3. Furthermore, the number of relays assisting each of the DMTs was assumed to be $L=0,1,2,3,4$, where $L=0$ corresponds to using no relays.

Observe from the results of Figs. 2 and 3 that the BER performance improves, when the number of relays increases, provided that the average SNR per bit is sufficiently high. By contrast, when the average SNR per bit is too low, as shown in Figs. 2 and 3, no useful relay diversity may be achieved and hence the BER performance might even degrade, when increasing the number of relays. Note that, this degradation is not a deficiency of the relay diversity scheme proposed in this contribution, but a common characteristic of relay-aided diversity schemes based on the amplify-andforward (AF) principle $[3,5]$. As demonstrated by the results of Figs. 2 and 3, the BER performance of the relay-aided DSCDMA using $m$-sequences is better than that of its counterpart using random sequences, implying that random sequences may suffer from more severe noise amplification than $m$-sequences. The performance loss due to using random sequences instead of $m$-sequences becomes explicit, when the relay-aided DSCDMA supports $K=27$ downlink users, as shown in Fig. 3 . Furthermore, in Fig. 2 and Fig. 3 we observe no error-floors either for $m$-sequences or for random sequences, implying that the TZF-assisted preprocessing is capable of efficiently suppressing the MUI both at the relays as well as at the DMTs.

\section{CONCLUSIONS}

In this contribution a relay-aided cooperation DS-CDMA downlink transmission scheme has been proposed and investigated. In the proposed cooperation scheme the downlink MUI is efficiently suppressed by the TZF-assisted preprocessing carried out at the BS. The transmitter preprocessing is not dependent on any CSI, which is often difficult to convey to the transmitter side. In the proposed scheme, the relays are free from MUI and require only low-complexity signal processing for forwarding information to their DMTs. The simulation 
results show that relay diversity can be achieved, after the MUI is suppressed by the TZF-assisted preprocessing. Our future research will concentrate on communications scenarios experiencing frequency-selective fading and inter-cluster interference associated with invoking advanced detection strategies at the DMTs.

\section{ACKNOWLEDGEMENT}

The authors would like to acknowledge with thanks the financial assistance from EPSRC of UK.

\section{REFERENCES}

[1] A. Sendonaris, E. Erkip, and B. Aazhang, "Increasing uplink capacity via user cooperation diversity," in Proc. IEEE Int. Symp. on Info. Theory, Cambridge, MA, Aug. 1998, p. 156.

[2] A. Sendonaris, E. Erkip, and B. Aazhang, "User cooperation diversitypart I and II," IEEE Trans. Wireless Commun, vol. 51, no. 11, Nov. 2003.

[3] P. A. Anghel and M. Kaveh, "Exact symbol error probability of a cooperative network in a Rayleigh-fading environment," IEEE Trans. Wireless Commun., vol. 3, no. 5, pp. 1416-1421, Sept. 2004.

[4] L.-L. Yang and H.-H. Chen, "Error probability of digital communications using relay diversity over Nakagami- $m$ fading channel," IEEE Trans. Wireless Commun., vol. 7, no. 5, pp. 1806-1811, May 2008.
[5] M. O. Hasna and M.-S. Alouini, "Harmonic mean and end-to-end performance of transmission systems with relays," IEEE Trans. Commun., vol. 52, pp. 130-135, Jan. 2004

[6] W. Fang, L.-L. Yang, and L. Hanzo, "Single-user performance of DSCDMA using relay diversity and power allocation,"IET Proc. Commun., vol. 2, no. 3, pp. 462-472, Mar. 2008.

[7] B. R. Vojcic and W. M. Jang, "Transmitter precoding in synchronous multiuser communications," IEEE Trans. Commun., vol. 46, pp. 13461355, Oct. 1998

[8] R. Esmailzadeh, E. Sourour, and M. Nakagawa, "Prerake diversity combining in time-division duplex CDMA mobile communications," IEEE Trans. Veh. Technol., vol. 48, pp. 795-801, May 1999.

[9] L.-U. Choi and R. D. Murch, "Transmit-preprocessing techniques with simplified receivers for the downlink of MISO TDD-CDMA systems," IEEE Trans. Veh. Technol., vol. 53, pp. 285-295, Mar. 2004.

[10] L.-L. Yang, "A zero-forcing multiuser transmitter preprocessing scheme for downlink communications," IEEE Trans. Commun., vol. 56, no. 6 , pp. 862-865, June 2008

[11] L.-L. Yang, "Design linear multiuser transmitters from linear multiuser receivers," in Proc. IEEE International Conference on Communications, ICC'07, pp. 5258-5263, 24-28 June 2007.

[12] J. G. Proakis, Digital Communications (3rd edition.) McGraw Hill, 1995.

[13] N. Nakagami, "The $m$-distribution, a general formula for intensity distribution of rapid fading," in Statistical Methods in Radio Wave Propagation, W. G. Hoffman, ed. Oxford, England: Pergamon, 1960.

[14] L.-L. Yang and L. Hanzo, "Performance of generalized multicarrier DSCDMA over Nakagami-m fading channels," IEEE Trans. Commun., vol. 50, no. 6, pp. 956-966, June 2002. 